\title{
Distinguishable Prognostic Signatures of Left- and Right-Sided Colon Cancer: a Study Based on Sequencing Data
}

\author{
Liang Liang $^{\mathrm{a}} \quad$ Jiang-hui Zeng ${ }^{\mathrm{b}}$ Xin-gan Qin ${ }^{\mathrm{a}} \quad$ Jun-qiang Chen ${ }^{\mathrm{a}}$ \\ Dian-zhong Luo ${ }^{\mathrm{b}}$ Gang Chen ${ }^{\mathrm{b}}$ \\ aDepartment of Gastrointestinal Surgery, First Affiliated Hospital of Guangxi Medical University, \\ Nanning, 'Department of Pathology, First Affiliated Hospital of Guangxi Medical University, Nanning, \\ China
}

\section{Key Words}

Left- and right-sided $•$ Colon cancer $•$ Prognosis $•$ TCGA

\begin{abstract}
Background/Aims: Left- and right-sided colon cancers are considered to be two different diseases and have altered outcomes. However, specific molecules to predict the prognosis of left- and right-sided colon cancers are currently lacking. Methods: Expression profiling of colon cancer were downloaded from The Cancer Genome Atlas (TCGA). Differentially expressed genes (DEGs) of left- and right-sided colon cancers were compared by DESeq analysis. The prognostic values of DEGs were assessed by univariate and multivariate Cox regression. Prognostic index models of two side colon cancers were conducted with prognostic values genes, respectively. Interaction of DEGs was then analyzed by the protein-protein interaction (PPI). Different biology function of two sides of colon cancer was assessed by Gene Set Enrichment Analysis (GSEA). Results: A total of 167 DEGs were identified between left- and right-sided colon cancers based on TCGA data. Using univariate COX regression analysis, five genes (PHACTR3, CKMT2, CYP2W1, ERFE, HOXC4) were related to overall survival in left-sided, and eight distinguishable genes (EREG, ERFE, HOXC6, SLC22A31, TFF1, GFI1, ZG16, RASL10B) in right-sided. Further, left-sided prognostic model was established with PHACTR3 and CKMT2 $(\mathrm{HR}=2.040 ; 95 \% \mathrm{CI}=1.004-4.145 ; \mathrm{P}=0.049)$. Distinguishable prognostic signature for right-sided colon cancer was established based on EREG, ERFE, GFI1, and RASL10B (HR=3.530; 95\%CI: 1.934-6.444; $\mathrm{P}<0.001$ ) in multivariate analysis. PPI analysis of $167 \mathrm{DEGs}$ showed that CCL5, GNG4, GNLY, GZMH, DRD2, and FASLG genes were at the core of interaction network. In GSEA function analysis, four pathways, including antigen processing and presentation, natural killer cell mediated cytotoxicity, intestinal immune network for Iga production, and type I diabetes mellitus, were significantly enriched in the DEGs of the right-sided colon cancer. Conclusions: This study constructs a panel of potential prognostic model of left- and right-sided colon cancers, respectively. We also provide molecular biological alterations between left- and rightsided colon cancers.




\section{Cellular Physiology Cell Physiol Biochem 2018;48:475-490 \begin{tabular}{c|c} 
DOI: 10.1159/000491778 & O 2018 The Author(s). Published by S. Karger AG, Basel \\
www.karger.com/cpb
\end{tabular} \\ Liang et al.: Prognostic Signatures in Left- and Right-Side Colon Cancer}

\section{Introduction}

Colon cancer has the enormously high rate among the cancers of the digestive system and is currently the third leading cause of morbidity and mortality worldwide [1-5]. Studies have verified that left- and right-sided colon cancers differ in embryonic origin, anatomy, physiology, and pathological type, as well as gender, age and other demographic characteristics [6-9]. In 1990, for the first time, Bufill discussed the alterations between left- and right-sided colon cancers regarding embryonic origin and genetic background. The right-sided colon comprises the ileocecum, the ascending colon, and the transverse colon. The left-sided colon contains the descending colon and the sigmoid colon [6]. According to the 2011 Surveillance, Epidemiology and End Results (SEER) database of the US National Cancer Institute, the occurrence of right-sided colon cancer increased by $25.26 \%$ during the 30 years from 1976 to 2005, with a mean annual growth rate of 0.68\% [10]. Yahagi M et al. investigated the variation of the prognosis of left- and right-sided colon cancers using a systematic retrospective review and meta-analysis. Their results revealed that the location of the tumor was correlated with survival and prognosis, and the overall survival of rightsided colon cancer was pronouncedly poorer than that of left-sided colon cancer [11]. The National Comprehensive Cancer Network (NCCN) Clinical Practice Guidelines in Oncology clearly declare that the therapeutic sensitivity of molecular targeted drugs differs for tumors in different locations. Treatment with EGFR inhibitors, such as cetuximab, panitumumab, or irinotecan can significantly prolong the survival of patients with left-sided colon cancer [12]. For RAS wild-type colon cancer, left-sided tumors had longer overall survival than rightsided tumors treated with bevacizumab. It may be due to the random distribution of various molecular subtypes in the colon, and the biological differences between right and left colon that cause distinct efficacy of molecularly targeted agent in patients with left and right colon [13]. In recent years, molecular biology techniques and high-throughput sequencing technology further promoted the study of the alterations between left- and right-sided colon cancers $[14,15]$. However, specific molecules that predict the prognosis of left- and rightsided colon cancers are currently lacking.

In this study, sequencing data from The Cancer Genome Atlas (TCGA) database were used to analyze differentially expressed molecules in left- and right-sided colon cancers and their predictive power for prognosis. We aimed to identify effective prognostic factors for left- and right-sided colon cancers and preliminary investigate their mechanisms, thereby contributing to the search for new clinical therapeutic targets for left- and right-sided colon cancers. The present study was summarized in Fig. 1.

\section{Materials and Methods}

Colon cancer samples and clinical parameter downloading

SEER. The SEER database (https://seer.cancer.gov/) was used to download the clinical information and survival status of patients with colon cancer from 1988 to 2010 for univariate analysis. Patients were disqualified if the information of tumor location and the T stage was missing. Cases with unclear tumor location, survival time and survival status were excluded.

TCGA. The colon adenocarcinoma sample information, the RNA-seq of transcriptome profiling count data, and the clinical data of the samples in TCGA were downloaded through the GDC data portal. The miRNAs data were downloaded from TCGA project, which provide the expression of precursor miRNAs. Cases with incomplete prognostic information were excluded.

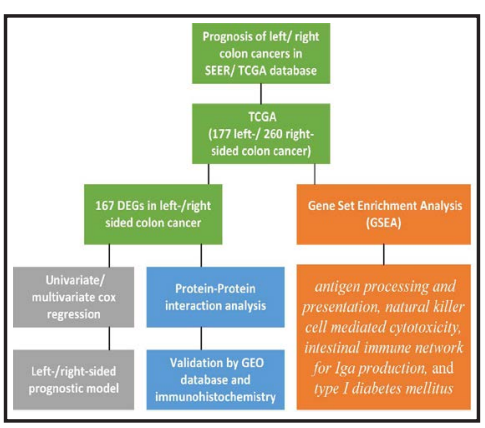

Fig. 1. The flow chart of present study. 


\section{Cellular Physiology Cell Physiol Biochem 2018;48:475-490 \begin{tabular}{l|l|l} 
and Biochemistry $10.1159 / 000491778$ & $\begin{array}{l}\text { DO } 2018 \text { The Author(s). Published by S. Karger AG, Basel } \\
\text { www.karger.com/cpb }\end{array}$
\end{tabular} \\ Liang et al.: Prognostic Signatures in Left- and Right-Side Colon Cancer}

Differently transcriptional level of left- and rightsided colon cancers

According to the primary site of the colon tumor, this study included 258 patients of right-sided colon cancer (ileocecum, ascending colon, hepatic flexure of the colon, and transverse colon) and 180 patients of left-sided colon cancer (splenic flexure of the colon, descending colon, and sigmoid colon) (Table 1). Differential expressions various molecules of mRNA, lncRNA and miRNA of left- and rightsided colon cancers were computed using the DESeq method. Genes with an expression value of 0 for greater than $10 \%$ of the expression were excluded; $|\log \mathrm{FC}|>1$ and adjust-P value $<0.05$ served as the screening conditions for significant difference. The log2 of the standard expression was used for subsequent analysis.

Prognostic factors of left- and right-sided colon cancers and construction of Cox regression models

To explore the different prognostic molecules of leftand right-sided colon cancers, the impact of the different molecular expression levels on the prognosis of patients with left- and right-sided colon cancers was investigated by univariate Cox regression analysis. The molecules that affect the survival of the patients with left- and rightsided colon cancers were preliminarily screened. Multiple stepwise Cox regression analysis was performed for potentially relevant genes in the preliminary screening, and the impact of these molecules on the survival time and the survival outcome was also analyzed. The screened molecules were used to construct the prognostic index (PI) model: $\left(\beta_{1} \operatorname{Exp}_{1}+\beta_{2} \operatorname{Exp}_{2}+\ldots+\beta_{\mathrm{x}} \operatorname{Exp}_{\mathrm{x}}\right)$ [16-21]. The larger the PI was, the greater the patient's risk and the worse the prognosis. The median of PI was used to divide the PI into a high-risk group and a low-risk group for KM univariate analysis to determine the prognostic power in the high-risk group and the low-risk group. $\mathrm{P}<0.05$ served as a standard condition for the above screening.

\section{Functional analysis of left- and right-sided colon cancers}

To explore the variances in biological functions between the left-sided colon and the right-sided colon, the Gene Set Enrichment Assay (GSEA) method was used for the analysis in this study [22]. All genes with different expression levels were divided into the left-sided colon group and the right-sided colon group for comparison. GSEA does not focus on a few individual genes with a significant change in expression. Instead, GSEA focuses on the consistent expression of the entire hybridization data in a specific set of functional genes to interpret the biological information contained in the data. FDR $<0.05$ was used as the inclusion criterium in the screening. In addition, the interaction of the differential molecules between the left-sided and right-sided colon was investigated using the PPI interaction analysis of these differential genes.

Verification of the expression of prognostic molecules

The differential molecules were validated using the colon cancer microarray data set in the Gene Expression Omnibus (GEO) database. The microarray data that contained tumor site information and can distinguish between left- and right-sided colon cancers were included in the analysis. The microarray expression profile data were downloaded and normalized. Relevant gene expression data were obtained by re-annotating the probe. T-tests were performed for difference analysis, and $\mathrm{P}<0.05$ was used as the screening standard. We also researched the genetic alterations of prognosis-related genes in colon cancer by cBioPortal web software (http://www.cbioportal.org/).

Table 1. Clinical characteristic of left- and ight-sided colon cancer

\begin{tabular}{|c|c|c|c|c|}
\hline ID & Left colon( $\mathrm{n}=180$ & Right colon $(\mathrm{n}=258)$ & 난 & $P$ \\
\hline Age & & & 6.699 & 0.010 \\
\hline$>=65$ & $98(54.4 \%)$ & $172(66.7 \%)$ & & \\
\hline$<65$ & $82(45.6 \%)$ & $86(33.3 \%)$ & & \\
\hline Gender & & & 0.043 & 0.835 \\
\hline Male & $96(53.3 \%)$ & $135(52.3 \%)$ & & \\
\hline Female & $84(46.7 \%)$ & $123(47.7 \%)$ & & \\
\hline Stage & & & 6.900 & 0.075 \\
\hline I & $30(16.9 \%)$ & $44(17.7 \%)$ & & \\
\hline II & $60(33.9 \%)$ & $109(43.8 \%)$ & & \\
\hline III & $54(30.5 \%)$ & $68(27.3 \%)$ & & \\
\hline IV & $33(18.6 \%)$ & $28(11.2 \%)$ & & \\
\hline Pathologic M & & & 4.448 & 0.035 \\
\hline M0 & $131(80.4 \%)$ & 194(88.2\%) & & \\
\hline M1 & $32(19.6 \%)$ & $26(11.8 \%)$ & & \\
\hline Pathologic N & & & 8.832 & 0.012 \\
\hline No & $96(53.3 \%)$ & $163(63.2 \%)$ & & \\
\hline N1 & $53(29.4 \%)$ & $45(17.4 \%)$ & & \\
\hline N2 & $31(17.2 \%)$ & $50(19.4)$ & & \\
\hline Pathologic T & & & 2.995 & 0.392 \\
\hline T1 & $4(2.2 \%)$ & $5(1.9 \%)$ & & \\
\hline T2 & $35(19.4 \%)$ & $42(16.3 \%)$ & & \\
\hline T3 & $125(69.4 \%)$ & $174(67.7 \%)$ & & \\
\hline T4 & $16(8.9 \%)$ & $36(14.0 \%)$ & & \\
\hline Venous invasion & & & 0.253 & 0.615 \\
\hline Yes & $40(25.3 \%)$ & $51(23.1 \%)$ & & \\
\hline No & $118(74.7 \%)$ & $170(76.9 \%)$ & & \\
\hline Lymphatic invasion & & & 2.720 & 0.099 \\
\hline Yes & $72(44.2 \%)$ & $83(35.9 \%)$ & & \\
\hline No & $91(55.8 \%)$ & $148(64.1 \%)$ & & \\
\hline Histological & & & 12.599 & $<0.001$ \\
\hline Adenocarcinoma & $166(93.3 \%)$ & $209(81.3 \%)$ & & \\
\hline Mucinous adenocarcinoma & $12(6.7 \%)$ & $48(18.7 \%)$ & & \\
\hline Colon polyps & & & 1.956 & 0.162 \\
\hline Yes & $22(28.9 \%)$ & $52(38.5 \%)$ & & \\
\hline No & $54(71.1 \%)$ & $83(61.5 \%)$ & & \\
\hline Chemotherapy & & & 1.989 & 0.158 \\
\hline Yes & $66(36.7 \%)$ & $78(30.2 \%)$ & & \\
\hline No & $114(63.3 \%)$ & $180(69.8 \%)$ & & \\
\hline Treatment outcome & & & 0.159 & 0.690 \\
\hline $\mathrm{SD}+\mathrm{PD}$ & $19(20.0 \%)$ & $24(17.9 \%)$ & & \\
\hline $\mathrm{CR}+\mathrm{PR}$ & $76(80.0 \%)$ & $110(82.1 \%)$ & & \\
\hline Residual tumor & & & 7.016 & 0.020 \\
\hline R0 & $132(89.2 \%)$ & $199(93.4 \%)$ & & \\
\hline R1 & $0(0.0 \%)$ & $4(1.9 \%)$ & & \\
\hline R2 & $16(10.8 \%)$ & $10(4.7 \%)$ & & \\
\hline Cancer status & & & 6.548 & 0.011 \\
\hline With tumor & $98(60.5 \%)$ & $109(47.4 \%)$ & & \\
\hline Tumor free & $64(39.5 \%)$ & $121(52.6 \%)$ & & \\
\hline Microsatellite instability & & & 3.424 & 0.064 \\
\hline Yes & $1(2.9 \%)$ & $10(18.9 \%)$ & & \\
\hline No & $33(97.1 \%)$ & $43(81.1 \%)$ & & \\
\hline Kras mutation & & & 0.123 & 0.726 \\
\hline Yes & $8(42.1 \%)$ & $10(47.6 \%)$ & & \\
\hline No & $11(57.9 \%)$ & $11(52.4 \%)$ & & \\
\hline BRAF mutation & & & 1.795 & 0.529 \\
\hline Yes & $0(0.0 \%)$ & $3(17.6 \%)$ & & \\
\hline No & $9(100.0 \%)$ & $14(82.4 \%)$ & & \\
\hline
\end{tabular}




\section{Cellular Physiology Cell Physiol Biochem 2018;48:475-490

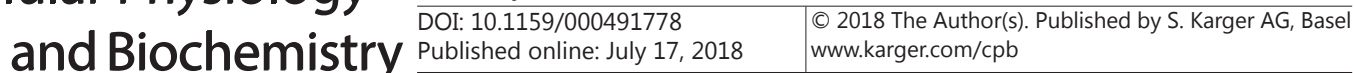

Liang et al.: Prognostic Signatures in Left- and Right-Side Colon Cancer

Meta-analyses of the expression levels of the hub genes from PPI analysis

We performed meta-analyses of the expression levels of PPI hub genes (CCL5, GNG4, GNLY, GZMH, DRD2, and FASLG) based on data extracted from microarray and RNA-seq databases. All data were converted by corresponding standardization and logarithm 2. Continuous variable expression levels were sent for the calculation of Standard Mean Difference (SMD) by STATA 12. Because of the heterogeneity of different studies, the random effect model was chosen. When the pooled SMD $<0$, the gene expression in left colon cancer was higher than that in right colon cancer; when SMD was $>0$, the gene expression was higher in right colon cancer than that in left colon cancer.

\section{Immunohistochemistry staining and evaluation of a hub gene}

To validate the protein level of the hub genes, one gene was selected as an example. We examined the expression of GNG4 protein levels by immunohistochemistry. The study was approved by the Ethical Committee of the first affiliated hospital of Guangxi Medical University and written informed consent was obtained from all patients. Forty cases of colon cancers (left side, $n=20$, right side, $n=20$ ) were collected in the first affiliated hospital of Guangxi Medical University from 2016 to 2017. The samples were fixed by formalin. The first antibody (bs-13245R, Anti-G gamma4, Bioss, Beijing, China) was diluted to a concentration (1:500) and incubated at $37^{\circ} \mathrm{C}$ for one hour. The secondary antibody was incubated at room temperature for half an hour. The immunohistochemistry results were reviewed by two independent pathologists (Jiang-hui Zeng and Gang Chen). We quantified the results using the immunoreactivity score system. The staining intensity ranged from 0 to 3, indicating no staining, weak staining, moderate and strong staining, respectively. The percentage of staining consists of $0-4$ points, 0 was $<5 \%$; 1 was $<5-25 \%$; 2 was $25-50 \%$; 3 was $51-75 \%$; 4 was $>75 \%$. The final result of each case was obtained by a score of multiplying the above two elements. The paired sample T-test was used to assess the difference of GNG4 expression between two groups. $\mathrm{P}<0.05$ was considered statistically significant.

\section{Results}

Prognosis of left- and right-sided colon cancers in SEER and TCGA

We divided SEER colon cancer cases into left- and right-sided colon cancers. A total of 572, 265 colon cancer patients were collected from 1988 to 2010, including 324, 827 rightsided colon cancer and 247, 438 left-sided colon cancer cases. The K-M univariate analysis results indicated that the overall survival rate of left-sided colon cancer was more favorable than that of right-sided colon cancer (log rank, $\mathrm{P}<0.001$, Fig. $2 \mathrm{~A}$ ). The median survival of left-sided colon cancer was 75 months (74.289-75.711 months), and the median survival of right-sided colon cancer was 60 months (59.493-60.507 months). In addition, the prognosis of left-sided colon cancer was enhanced compared with that of right-sided colon cancer at different tumor stages (log rank, $\mathrm{P}<0.001$, Fig. 2B, C).

Furthermore, K-M prognostic analysis was performed on 170 cases of left-sided colon cancer and 251 cases of right-sided colon cancer using TCGA. No remarkable alteration in overall survival was noted between the left- and right-sided colon cancers, as shown in Fig. $3 \mathrm{~A}$ ( $\log$ rank, $\mathrm{P}=0.064$ ). However, our analysis of patients at different tumor stages revealed that among patients with high-stage colon cancer, better prognosis was associated with leftsided colon cancer compared with right-sided colon cancer (Log Rank, P = 0.030, Fig. 3B). For those patients who were in the early stage, the prognoses of the left-sided and rightsided colon cancers did not change noticeably (Fig. 3C).

\section{Differential molecules in left- and right-sided colon cancers}

For the TCGA data, differences in gene expression were analyzed using the DESeq method by dividing colon cancer cases into left- and right-sided colon cancers. A total of 167 differential genes met the criteria of fold change $>2$ and adj-P value $<0.05$, including 109 genes (containing 8 lncRNAs) highly expressed in right-sided colon cancer and 58 genes (containing 6 lncRNAs) highly expressed in left-sided colon cancer, with the most evidential alteration noted in GNG4 (Fig. 4, Table 2). A total of 325 miRNAs could be calculated between 


\section{Cellular Physiology Cell Physiol Biochem 2018:48:475-490

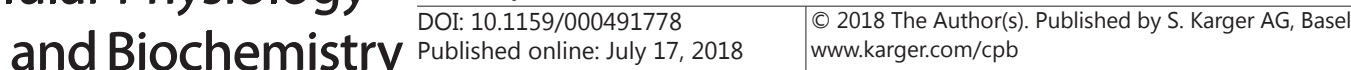 \\ Liang et al.: Prognostic Signatures in Left- and Right-Side Colon Cancer}

left- and right-sided colon cancers. Only miR-592 was differentially expressed between leftand right-sided colon cancers (data not shown).

Fig. 2. The survival of patients with left- or rightsided colon cancer based on SEER database. Kaplan-Meier (K-M) survival analysis was showed the correlation between survival and tumor location. (A) Left colon cancer had a better survival than right colon cancer (log-rank, $\mathrm{P}<0.001)$. (B) In tumor stage I+II, patients with left colon cancer showed longer survival than right colon cancer (log-rank, $\mathrm{P}<0.001$ ). (C) In advanced tumor stage, survival time of patients with left colon cancer is prolonged than right colon cancer (log-rank, $\mathrm{P}<0.001$ )

Fig. 3. The survival of patients with left- or rightsided colon cancer based on TCGA database. Kaplan-Meier (K-M) survival analysis was showed the correlation between survival and tumor location. (A) Survival time of patients with left- and right-sided colon cancers was not different. (logrank, $\mathrm{P}=0.064$ ). (B) Survival time of patients with left- and right-sided colon cancer was not different in early stage of tumor. (log-rank, $\mathrm{P}=0.395$ ) (C) In advanced tumor stage, survival time of patients with left colon cancer is prolonged than right colon cancer (log-rank, $\mathrm{P}=0.030$ )

Fig. 4. Differentially expressed genes between left- and right-sided colon cancer. (A) The volcano plot showed 167 differentially genes calculated by DESeq method with criteria $(\log 2 \mathrm{FC}>1$, adj- $\mathrm{P}<0.05)$. The red dots represented the up expressed genes, as well as green were down expressed genes. (B) Hierarchical clustering of differentially expressed genes expression levels.

Prognosis models of left-sided and rightsided colon cancers

In TCGA, univariate Cox regression analysis was performed for 167 DEGs in leftand right-sided colon cancers to identify five prognostic genes for left-sided colon cancer, including PHACTR3, CKMT2, CYP2W1, ERFE and HOXC4, and eight prognostic genes for the right-sided colon cancer, including EREG, ERFE, HOXC6, SLC22A31,
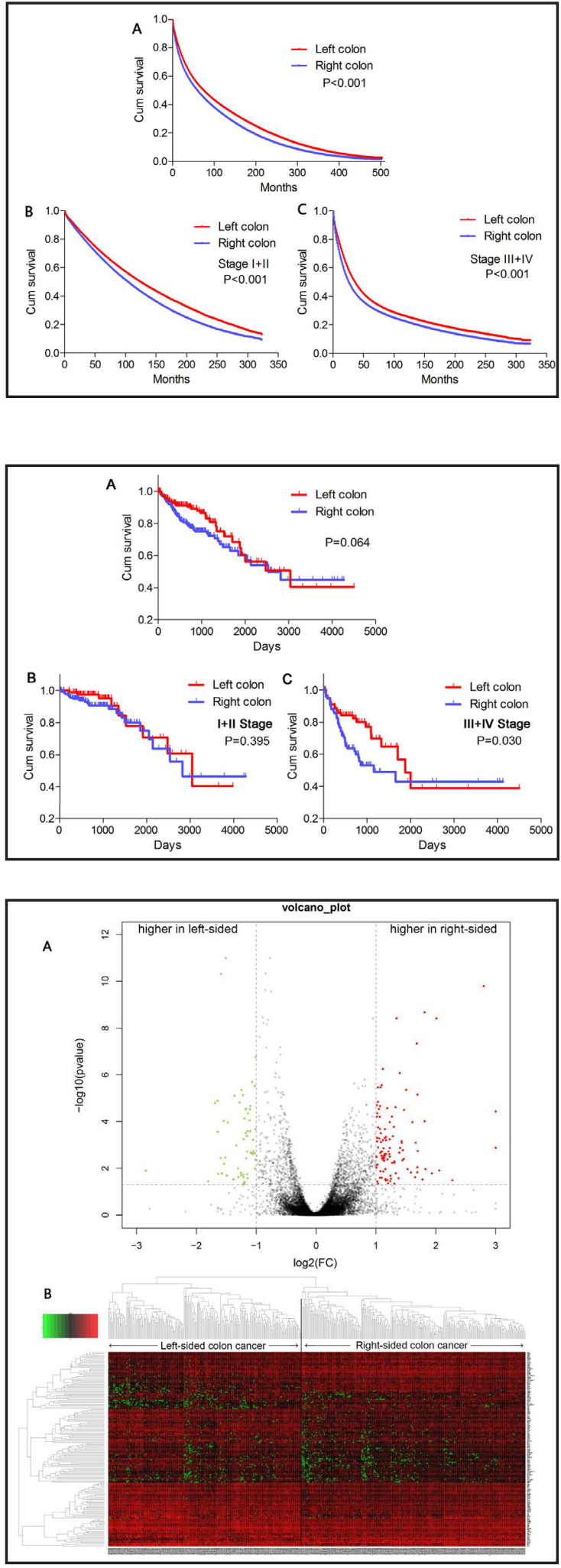
TFF1, GFI1, ZG16, and RASL10B (Table 3). Multiple stepwise Cox regression was used to analyze the effect of five left-sided colon cancer RNAs on the survival time and the patient's outcome. PHACTR3 and CKMT2 genes affected the survival time and the patient's outcome (Fig. 5). A prognostic index model for left-sided colon cancer was constructed using these two genes. The expression data of these two genes were introduced into the PI equation, PI $=0.299 \times$ PHACTR $_{\text {EXP }}-0.174 \times$ CKMT2 $_{\text {EXP' }}$ obtaining PI for the 170 cases (Fig. 6). Similarly, multiple stepwise Cox regression was performed for the eight genes for right-sided colon cancer, including EREG, ERFE, HOXC6, SLC22A31, TFF1, GFI1, ZG16 and RASL10B. The results revealed that EREG, ERFE, GFI1, and RASL10B were

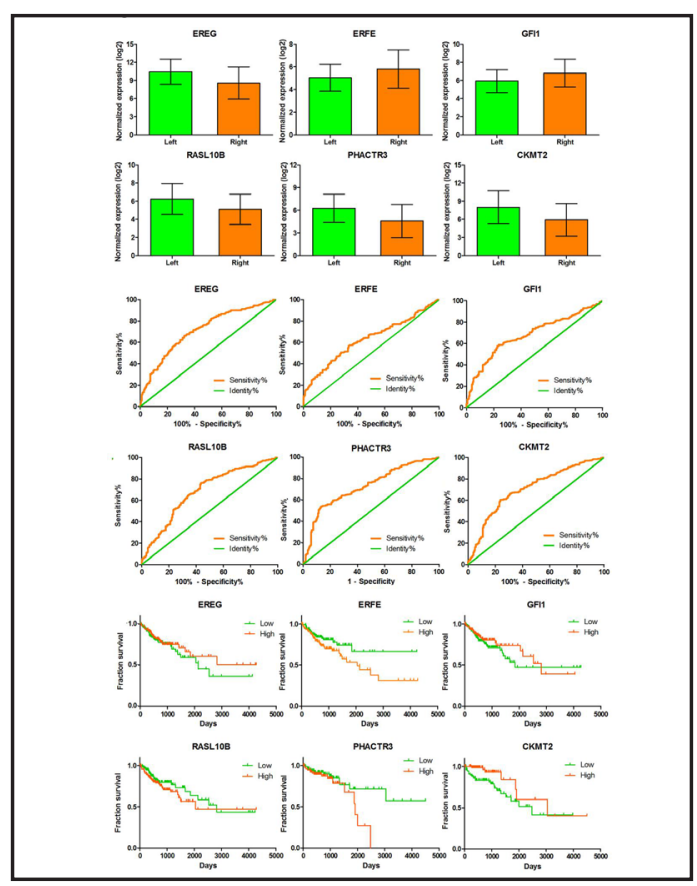

Fig. 5. Clinical parameters of 6 genes of prognostic index model. EREG, RASL10B, PHACTR3 and CKMT2 were significantly highly expressed in left-sided colon cancer in TCGA. Besides, ERFE and GFI1 were lower expression in left-sided than right-sided colon cancer. ROC curve analysis showed that EREG, ERFE, GFI1, and RASL10B had high diagnostic value in right-sided colon cancer. PHACTR3 and CKMT2 also had high significant diagnostic values in left-sided colon cancer. Kaplan-Meier survival analysis was divided low and high expression group as medium cut-off value.
Table 2. 167 differentially expressed genes between left- and right-sided colon cancer

\begin{tabular}{|c|c|c|c|c|c|}
\hline & & & & & 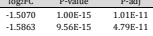 \\
\hline 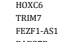 & 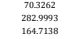 & 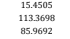 & 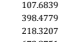 & 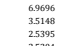 & 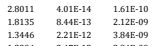 \\
\hline 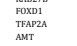 & 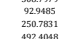 & 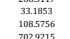 & 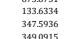 & 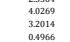 & 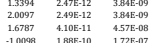 \\
\hline 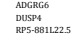 & 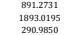 & 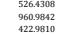 & 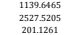 & 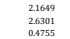 & 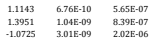 \\
\hline 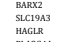 & 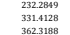 & 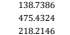 & 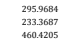 & 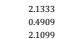 & 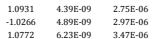 \\
\hline 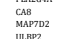 & 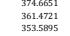 & 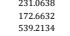 & 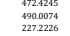 & 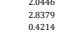 & 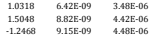 \\
\hline 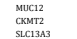 & 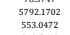 & 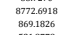 & 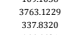 & 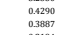 & 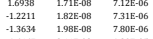 \\
\hline 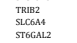 & 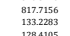 & 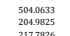 & 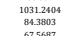 & 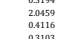 & 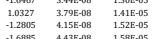 \\
\hline & 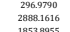 & 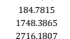 & & $\begin{array}{lll}202025 \\
\text { 201050 } \\
\text { ans }\end{array}$ & 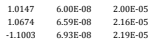 \\
\hline 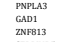 & 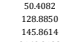 & 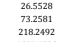 & 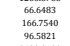 & 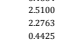 & 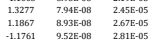 \\
\hline 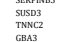 & 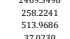 & 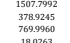 & 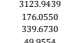 & 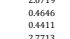 & 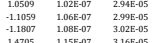 \\
\hline 业 & 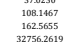 & 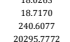 & 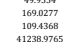 & 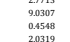 & 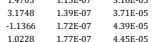 \\
\hline (a) & 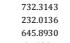 & 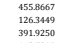 & 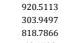 & 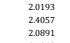 & 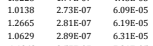 \\
\hline & 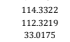 & 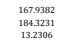 & 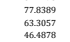 & 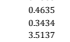 & 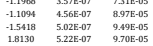 \\
\hline 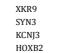 & 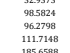 & 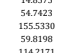 & 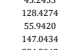 & 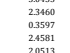 & 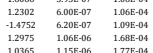 \\
\hline 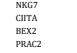 & 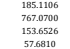 & 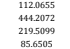 & 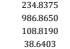 & 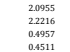 & 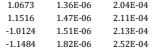 \\
\hline 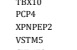 & 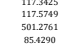 & 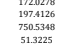 & 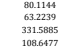 & 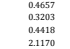 & 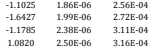 \\
\hline 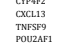 & 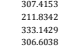 & 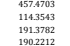 & 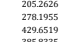 & 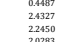 & 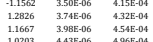 \\
\hline 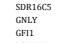 & 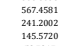 & 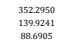 & 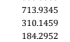 & 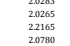 & 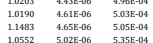 \\
\hline 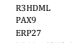 & 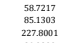 & 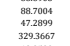 & 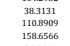 & 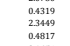 & 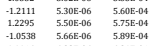 \\
\hline 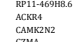 & 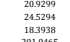 & 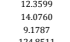 & 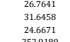 & 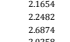 & 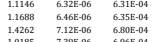 \\
\hline 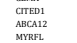 & 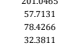 & 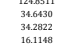 & 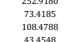 & 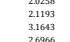 & 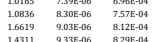 \\
\hline 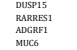 & 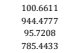 & 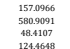 & 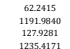 & 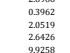 & 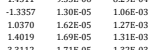 \\
\hline 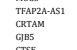 & 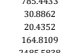 & 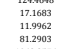 & 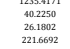 & 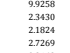 & 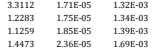 \\
\hline 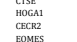 & 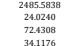 & 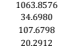 & 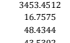 & 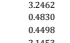 & 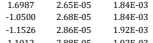 \\
\hline 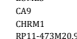 & 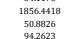 & 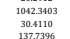 & 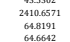 & 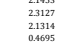 & 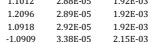 \\
\hline tarpus & 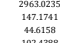 & 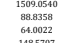 & 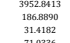 & 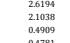 & 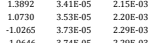 \\
\hline 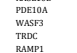 & 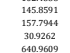 & 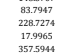 & 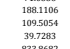 & 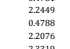 & 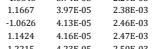 \\
\hline & 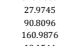 & 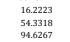 & 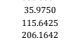 & 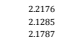 & 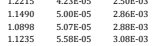 \\
\hline tats & 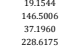 & 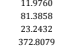 & 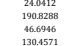 & 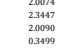 & 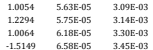 \\
\hline 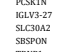 & 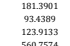 & 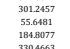 & 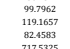 & 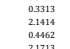 & 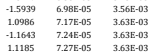 \\
\hline 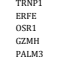 & 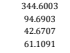 & 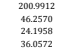 & 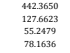 & 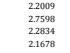 & 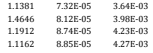 \\
\hline & 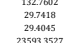 & 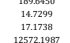 & 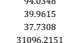 & 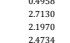 & 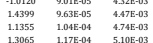 \\
\hline 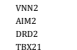 & 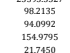 & 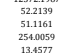 & 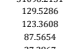 & 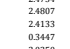 & 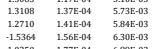 \\
\hline tation & 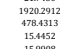 & 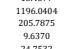 & 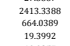 & 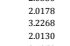 & 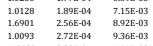 \\
\hline & 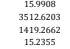 & 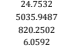 & 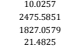 & 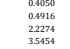 & 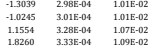 \\
\hline & 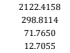 & 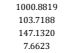 & 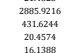 & 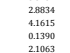 & 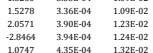 \\
\hline 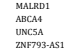 & 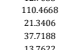 & 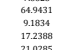 & 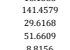 & 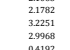 & 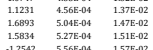 \\
\hline & 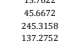 & 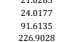 & 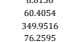 & 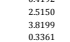 & 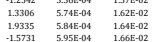 \\
\hline & 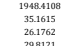 & 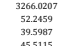 & 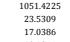 & 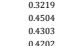 & 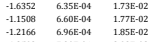 \\
\hline & 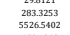 & 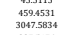 & 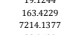 & 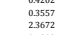 & 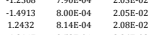 \\
\hline 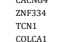 & 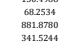 & 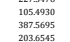 & 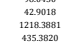 & 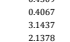 & 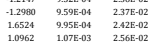 \\
\hline 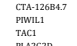 & 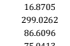 & 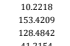 & 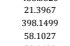 & 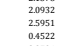 & 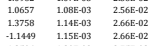 \\
\hline & 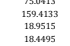 & 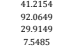 & 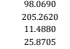 & 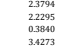 & 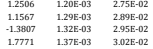 \\
\hline & 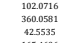 & 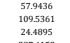 & 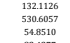 & 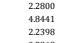 & 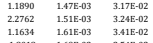 \\
\hline & 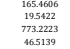 & 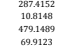 & 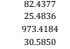 & 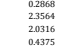 & 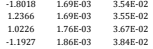 \\
\hline & 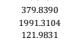 & 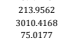 & 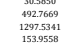 & 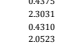 & 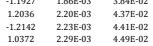 \\
\hline & & 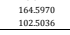 & & & \\
\hline
\end{tabular}


prognostic factors (Fig. 5). The expression data of these four genes were introduced into the prognostic index model for rightsided colon cancer, PI $=-0.125 \times \mathrm{EREG}_{\mathrm{EXP}}+$ $0.155 \times$ ERFE $_{\text {EXP }}-0.172 \times$ GFI1 $_{\text {EXP }}+0.149 \times$ RASL10B $_{\text {EXP }}$ (Fig. 7). The included cases were assigned into a low-risk group and a highrisk group according to the median cutoff of the PI. The PI could be determined as independent prognostic factor according to univariate and multivariate cox regression (Table 4). The K-M prognostic analysis revealed that the prognosis of the low-risk group was better than the high-risk group for both left- and right-sided colon cancers. The diagnostic rate of the 5-year survival rate by the prognostic model constructed with the risk index was AUC $=0.692$ for left-sided colon cancer and AUC $=0.730$ for right-sided colon cancer, as shown in Fig. 8. However, the expression level of miR-592 was not associated with OS neither in leftsided, nor right-sided colon cancer by cox regression analyses.

Gene set enrichment analysis for leftsided and right-sided colon cancers

To better understand the differences in biological function between the left- and right-sided colons, gene set enrichment analysis (GSEA) was performed. Four pathways, including antigen processing and presentation, natural killer cell mediated cytotoxicity, intestinal immune network for Iga production, and type I diabetes mellitus, were significantly enriched in the right-sided colon, whereas no pathway was significantly enriched in the left-sided colon (Fig. 9, Table 5). In addition, the interaction between the DEGs of the left- and right-sided colon cancers was analyzed by the PPI interaction network. Fig. 10 indicated that the genes CCL5, GNG4, GNLY, GZMH, DRD2, and FASLG genes were more closely related to other genes, which may indicate an important role in regulation.

\section{GEO validation for the expression and genetic alterations of six prognostic genes}

A total of 20 microarray datasets were identified by

Table 3. Prognostic value of 167 genes of left- and rightsided colon cancer in univariate cox regression

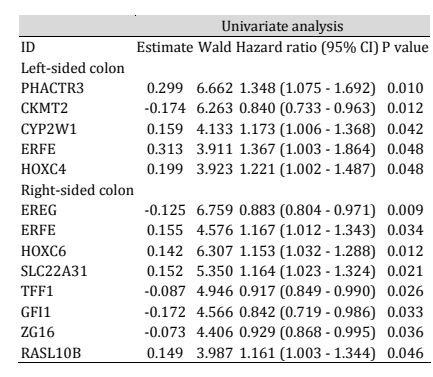
screening the GEO database. In some microarrays, the expression levels of PHACTR3 and CKMT2 for the left-sided colon prognosis model and EREG, ERFE, GFI1 and RASL10B for the right-sided colon prognosis model were verified. PHACTR3, CKMT2, EREG, GFI1 and RASL10B expression levels significantly differed between left- and the right-sided colon cancers, as shown in Fig. 11, which is consistent with the trend of expression noted in TCGA. As for genetic alterations, EREG showed the highest genetic alterations in these genes. ERFE and GFI1 did not show any alterations in colon cancer of TCGA (Fig. 12). 
Fig. 7. Prognostic index (PI) model of right-sided colon cancer. The top section showed the survival status of patients with right-sided colon cancer. Y-axis represented survival time of each patient. The middle section of Fig. showed the PI curve. X-axis represented the patient IDs, which were ranking by PI from low to high. The heatmap of bottom showed EREG, ERFE, GFI1 and RASL10B expression in each right-sided colon cancer patients.

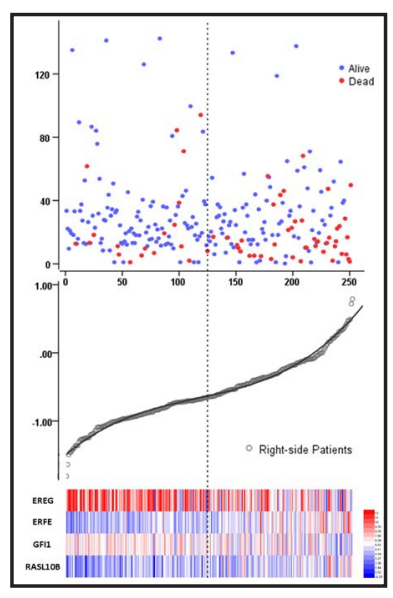

Fig. 8. The prognostic values of prognostic index (PI) of left- and right-sided colon cancer patients. (A) Kaplan-Meier (K-M) curve showed that low PI group had a better survival than high level PI group in right-sided colon cancer $(\mathrm{P}=0.004)$. (B) Time-dependent ROC curve represented 5-year survival prediction by the right-sided prognostic model (AUC=0.692). (C) Kaplan-Meier (K-M) curve showed that low PI group had a better survival than high level PI group in left-sided colon cancer $(\mathrm{P}<0.001)$. (D) Time-dependent ROC curve represented 5-year survival prediction by the left-sided prognostic model (AUC=0.730).

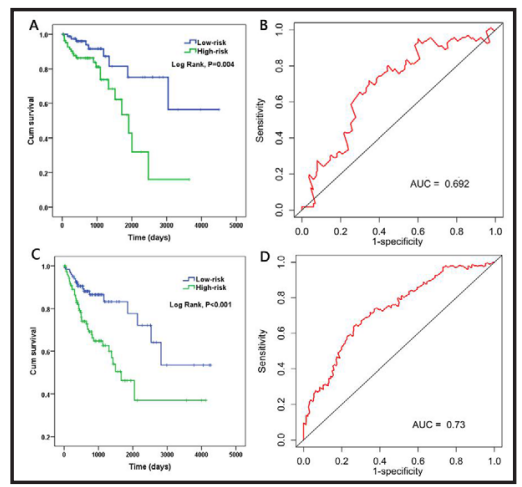

Meta-analyses of hub genes based on microarray and RNAseq data

According to 13 GEO data series and TCGA dataset, the results showed that GNG4, CCL5, GNLY, GZMH, DRD2 had the same dysregulated trends to our present study except FASLG. GNG4 (SMD=0.56, 95\%CI: $-0.87,-0.25, \mathrm{P}<0.001$ )

Table 4. Univariate and multivariate Cox analysis of risk score

\begin{tabular}{|c|c|c|c|c|c|c|c|c|}
\hline \multirow{3}{*}{ Left- } & \multicolumn{4}{|c|}{ Univariate analysis } & \multicolumn{4}{|c|}{ Multivariate analysis } \\
\hline & Estimate & e Wald & Hazard ratio $(95 \% \mathrm{Cl})$ & $\mathrm{P}$ value $\mathrm{E}$ & Estimate & Wald & Hazard ratio $(95 \% \mathrm{Cl})$ & Pvalu \\
\hline & & & & & & & & \\
\hline zel> & & & $1.141(0.553-2.354)$ & 0.722 & & & & \\
\hline & & 0.622 & & 0.430 & & & & \\
\hline & 1.234 & 8.750 & $3.437(1.517$ - 7.787) & 0.003 & 1.929 & 8.686 & $6.881(1.908-24.814)$ & 0.003 \\
\hline 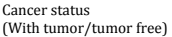 & 1.261 & 5.138 & $3.527(1.186-10.491)$ & 0.023 & 1.789 & 6.987 & $5.985(1.588-22.552)$ & 0.00 \\
\hline $\begin{array}{l}\text { prognostic index } \\
\text { Right-sided colon }\end{array}$ & 0.918 & 11.332 & $2.053(1.467-4.271)$ & $<0.001$ & 0.713 & 3.887 & $2.040(1.004-4.145)$ & 0.049 \\
\hline & & & & & & & & \\
\hline & & & & & & & & \\
\hline & 1.257 & 20.428 & $3.516(2.038$ - 6.066) & $<0.001$ & 1.403 & 18.251 & $4.066(2.136-7.738)$ & $<0.00$ \\
\hline & 1.236 & 15.442 & $3.443(1.859-6.380)$ & $<0.001$ & 0.946 & 8.151 & $2.574(1.345-4.927)$ & 0.004 \\
\hline prognostic index & 1.321 & 25.856 & $3.749(2.253-6.239)$ & $<0.001$ & 1.261 & 16.873 & $3.530(1.934-6.444)$ & $<0.00$ \\
\hline
\end{tabular}
and DRD2 (SMD $=-0.241,95 \%$ CI: -0.432 ,$0.05, \mathrm{P}=0.013$ ) were over-expressed in left-sided colon cancer than right one. CCL5 (SMD $=0.36,95 \% \mathrm{CI}: 0.21,0.51$, $\mathrm{P}<0.001)$, GNLY $(\mathrm{SMD}=0.294, \quad 95 \% \mathrm{CI}$ : $0.112, \quad 0.475, \quad \mathrm{P}=0.002)$, and GZMH (SMD $=0.308, \quad 95 \% \mathrm{CI}: \quad 0.199, \quad 0.417$, $\mathrm{P}<0.001)$ expression in right-sided were higher than in left-sided one (Fig. 13).

GNG4 expression of immunohistochemistry staining

GNG4 expression levels with 40 paired colon cancers were assessed by immunoreactive score as an example for

Table 5. KEGG pathways enrichment in GSEA analysis

\begin{tabular}{lccccc}
\hline NAME & SIZE & ES & NES & P-value & FDR \\
\hline Kegg_Natural_Killer_Cell_Mediated_Cytotoxicity & 103 & 0.562 & 1.933 & 0.006 & 0.033 \\
Kegg_Intestinal_Immun__Network_For_Iga_Production & 38 & 0.772 & 1.969 & 0.002 & 0.035 \\
Kegg_Antigen_Processing_And_Presentation & 56 & 0.718 & 1.997 & 0.006 & 0.037 \\
Kegg_Type_IDiabetes__ellitus & 36 & 0.755 & 1.938 & 0.000 & 0.040 \\
Kegg_Autoimmune__hyroid_Disease & 31 & 0.805 & 1.892 & 0.000 & 0.051 \\
Kegg_Viral_Myocarditis & 60 & 0.662 & 2.006 & 0.002 & 0.062 \\
Kegg_Graft_Versus_Host_Disease & 30 & 0.800 & 1.837 & 0.006 & 0.085 \\
Kegg_Allograft_Rejection & 30 & 0.797 & 1.822 & 0.006 & 0.088 \\
Kegg_Hematopoietic_Cell_Lineage & 72 & 0.623 & 1.781 & 0.028 & 0.117 \\
Kegg_Primary_Immunodeficiency & 30 & 0.660 & 1.745 & 0.022 & 0.151 \\
Kegg_Sphingolipid_Metabolism & 33 & 0.529 & 1.667 & 0.032 & 0.187 \\
Kegg_Asthma & 21 & 0.750 & 1.674 & 0.033 & 0.190 \\
Kegg_Chemokine_Signaling_Pathway & 168 & 0.468 & 1.691 & 0.031 & 0.195 \\
Kegg_Cytosolic_Dna_Sensing_Pathway & 40 & 0.502 & 1.651 & 0.038 & 0.198 \\
Kegg_Leishmania__nfection & 67 & 0.573 & 1.698 & 0.036 & 0.203 \\
Kegg_Prion_Diseases & 30 & 0.542 & 1.674 & 0.030 & 0.205 \\
Kegg_Colorectal_Cancer & 61 & 0.398 & 1.529 & 0.040 & 0.209 \\
Kegg_Long_Term_Potentiation & 59 & 0.437 & 1.608 & 0.020 & 0.211 \\
Kegg_Pancreatic_Cancer & 69 & 0.415 & 1.550 & 0.042 & 0.211 \\
Kegg_Apoptosis & 83 & 0.411 & 1.542 & 0.046 & 0.214 \\
Kegg_Toll_Like_Receptor_Signaling_Pathway & 83 & 0.469 & 1.613 & 0.046 & 0.215 \\
Kegg_T_Cell_Receptor_Signaling_Pathway & 101 & 0.440 & 1.618 & 0.040 & 0.220 \\
Kegg_B_Cell_Receptor_Signaling_Pathway & 73 & 0.453 & 1.622 & 0.049 & 0.226 \\
Kegg_Rig_I_Like_Receptor_Signaling_Pathway & 50 & 0.443 & 1.555 & 0.044 & 0.228 \\
Kegg_F__Epsilon_Ri_Signaling_Pathway & 69 & 0.415 & 1.562 & 0.044 & 0.235 \\
Kegg_Vegf_Signaling_Pathway & 68 & 0.406 & 1.569 & 0.016 & 0.236 \\
Kegg_Starch_And_Sucrose_Metabolism & 34 & 0.496 & 1.573 & 0.046 & 0.242 \\
\hline & & & & &
\end{tabular}




\section{Cellular Physiology Cell Physiol Biochem 2018;48:475-490 \begin{tabular}{l|l} 
DOI: 10.1159/000491778 & O 2018 The Author(s). Published by S. Karger AG, Basel \\
www.karger.com/cpb
\end{tabular}

Fig. 9. KEGG functional enrichment analysis of left- and right-sided colon cancer based on GSEA. (A) Four KEGG pathways (antigen processing and presentation, natural killer cell mediated cytotoxicity, intestinal immune network for Iga production, and type I diabetes mellitus) were significantly enriched in right-sided colon cancer (FDR $<0.05)$. (B) Heatmap of differentially expressed genes of left- and right-sided colon

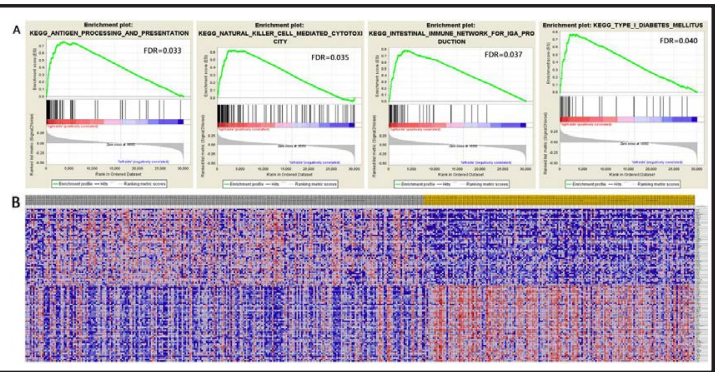
cancer.

Fig. 10. Protein-protein interaction (PPI) network of 167 differentially expressed genes of left- and right-sided colon cancer. PPI network showed the links between proteins. CCL5, GNG4, GNLY, GZMH, DRD2, and FASLG genes were identified as hub genes, which mostly connected with other genes. Medium confidence interaction score was set as cutoff. Disconnected nodes in the network were hided.

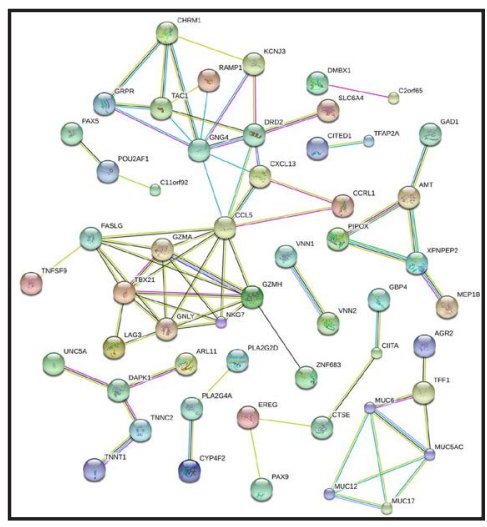

Fig. 11. Six genes expression of prognostic index model in GEO database. Six genes expression were validated in 14 selected data series. CKMT2 was over expressed in left colon cancer in 10 data series (GSE39084, GSE14333, GSE75316, GSE75315, GSE35834, GSE41258, GSE8671, GSE37892, GSE89076, and GSE50592). EREG was higher expression in left-sided than right-sided colon cancer (GSE39084, GSE14333, GSE75316, GSE75315, GSE35834, GSE41258, GSE77953, GSE44076, GSE72970, GSE103512). RASL10B, GFI1, PHACTR3 were showed differentially in other data series. Unpaired T-test was performed in analysis. $\mathrm{P}<0.05$ was set as significant.

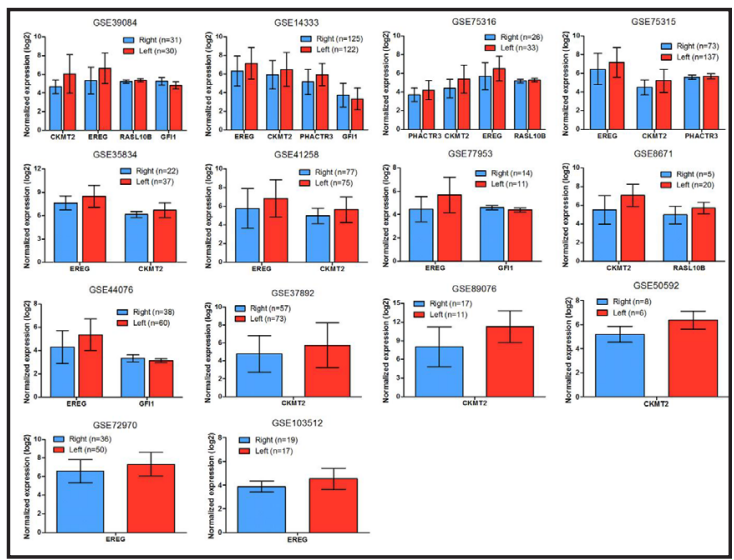

the validation of the hub genes. The expression level of GNG4 was prominently higher in colon cancer tissues (10.538 \pm 2.537$)$ compared with adjacent colon tissues $(2.589 \pm 2.152$, $\mathrm{P}<0.001)$. Furthermore, GNG4 expression in left colon cancer tissues $(11.600 \pm 1.231)$ were obviously higher than that in right colon cancer tissues $((9.850 \pm 2.815, \mathrm{P}=0.017$, Fig. 14).

\section{Discussion}

In the current study, a differential expression signature of various molecules was performed between the left-sided colon and the right-sided colon based on TCGA sequencing data including mRNA, IncRNA and miRNA, and the predictive power of these differential molecules on the survival of the patients with left- and right-sided colon cancers were further investigated. Molecules that could predict the prognosis of left- and right-sided colon cancers were separately identified, and the corresponding models of prognosis were constructed. 
Fig. 12. Genetic alterations of prognostic models. Based on TCGA, we used cBioPortal to detect Genetic alterations in 120 (30\% of total 394 ) colon cancers.

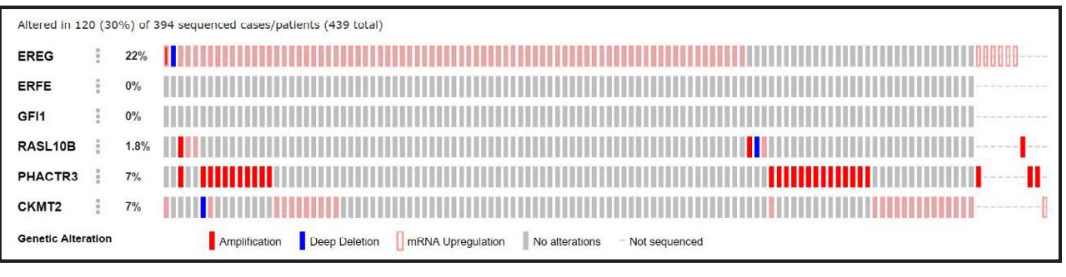

Fig. 13. Forrest plots of hub genes from PPI analysis. A: Forrest plot showed GNG4 overexpression in leftsided colon cancers; B: Forrest plot showed DRD2 overexpression in left-sided colon cancers; C: Forrest plot showed CCL5 overexpression in right-sided colon cancers; D: Forrest plot showed GNLY overexpression in right-sided colon cancers; E: Forrest plot showed GZMH overexpression in right-sided colon cancers; F: Forrest plot showed no statistical significance of FASLG expression in colon cancers.

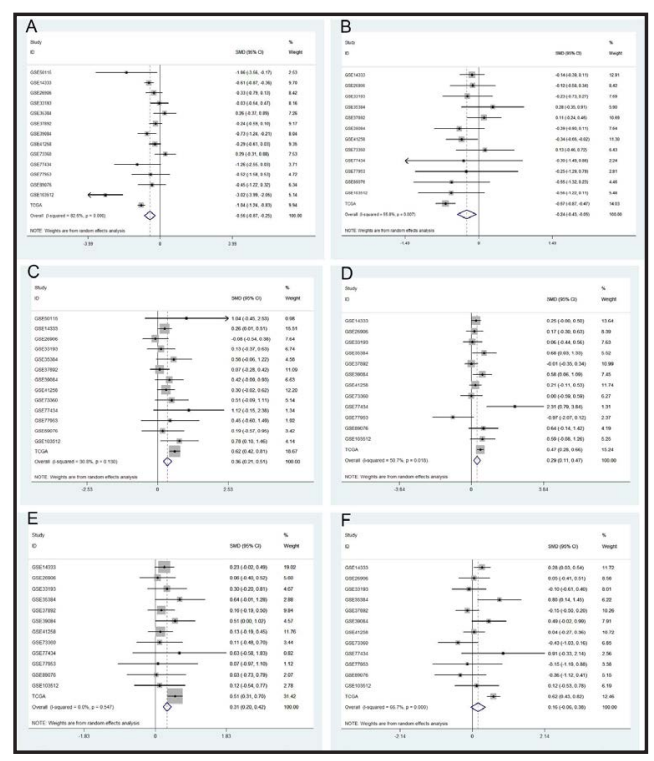

Prognostic index model of left-sided colon contained PHACTR3 and CKMT2, while another four genes (EREG, ERFE, GFI1 and RASL10B) were included in prognostic model of right-side colon cancer. An enrichment analysis of gene function was also carried out for the left- and the right-sided colon using GSEA. The results showed that right-sided colon cancer was related to the pathways of antigen processing and presentation, natural killer cell mediated cytotoxicity, intestinal immune network for Iga production, and type I diabetes mellitus. But the differentially expressed miRNAs and IncRNAs had no significant prognostic value between left- and right-sided colon cancers.

Some studies have focused on factors affecting the prognosis of the left and right colon cancer. Bauer et al. compared recurrence specimens of the left- and right-sided colon cancers and found that NOX4, CDX2, and ITGA3 expression levels differed. The reduced recurrence-free survival in left-sided colon cancer was related to high NOX4 expression. The reduced recurrence-free survival in rightsided colon cancer was related to high CDX2 expression $[23,24]$. Although they found some factors related to the prognosis of the left

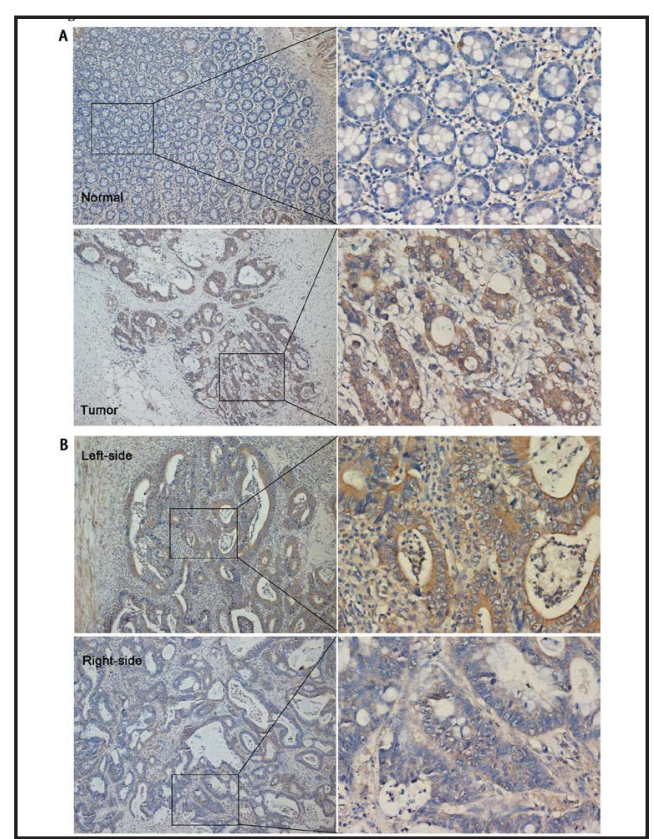

Fig. 14. GNG4 protein expression in left-/ right sided colon cancer. (A) GNG4 showed higher expression level in tumor tissue than normal tissue by IHC. (B) GNG4 showed higher expression level in left-sided colon cancer than right-sided colon cancer by IHC. Magnification: $\mathrm{x} 100$ and $\mathrm{x} 400$. 


\section{Cellular Physiology Cell Physiol Biochem 2018;48:475-490 \\ \begin{tabular}{l|l} 
DOI: 10.1159/000491778 & $\begin{array}{l}\text { O 2018 The Author(s). Published by S. Karger AG, Basel } \\
\text { www.karger.com/cpb }\end{array}$
\end{tabular} \\ Liang et al.: Prognostic Signatures in Left- and Right-Side Colon Cancer}

and right colon cancer, the predictive ability of a single gene may not be accurate, and the sample size was relatively small compared with the TCGA database. In the current study, a prognostic model to independently predict the prognosis of left-sided colon cancer was constructed using the differential genes PHACTR3 and CKMT2, and a prognostic model to independently predict the prognosis of right-sided colon cancer was constructed using four differential genes, including EREG, ERFE, GFI1 and RASL10B. Our GEO verification results revealed that PHACTR3, CKMT2, EREG, ERFE and RASL10B were highly expressed in the left-sided colon, and GFI1 was highly expressed in the right-sided colon, which is consistent with the aforementioned findings from TCGA dataset. Among the abovementioned genes, the PHACTR protein family is closely related to cell motivity and morphogenesis by regulating the actin cytoskeleton [25]. PHACTR3 is a new hypermethylated gene in CRC exhibiting good performance in stool DNA testing and has complementary value to the fecal immunochemical test in colorectal cancer [26]. Hypermethylation of the PHACTR3 promoter measured in stool samples could be used as a colorectal cancer biomarker and provide prognostic information [27]. CKMT2 was not studied in colon cancer. Wang $\mathrm{H}$ et al. validated that CKMT2 might serve as a key regulating factor participating in osteosarcomagenesis [28]. Saridaki Z et al. demonstrated that OS was significantly reduced in CRC patients with down-regulated EREG (HR: 1.7, P = 0.021), which was revealed as an independent prognostic factor for decreased OS [29]. Qu X et al. found that in those patients treated with cetuximab, low EREG methylation was correlated with increased expression of the ligand of EREG, also was related to a better response to the cetuximab treatment. On the contrary, high EREG methylation was correlated with decreased expression of the ligand of EREG, as well as was related to the worse response of therapy [30]. Lee MS et al. found that there were noteworthy inverse correlations between EREG expression and methylation, as well as tumor position, BRAF mutation, and CIMP-high status [31]. Inoue A et al. found that methylated RASL10B could be involved in the development from sessile serrated adenomas/polyps (SSA/Ps) to colon cancer [32]. Zou H et al. demonstrated that profound down-regulation of RASL10B mRNA level could be observed in all breast cancer cell lines and suggested that RASL10B could act as a novel member of the Ras superfamily, which owns the potential to be a tumor suppressor [33]. Xing W et al. explored that GFI1 could act as a gating modulator, which shoulders the responsibility for an essentially activated signaling circuit that leads to the metastasis of CRC [34]. Xing W et al. investigated that GFI1 could be a prospective therapeutic goal to hinder the progress and metastasis of inflammation-related CRC. Relapse-free survival (RFS) in cases with increased GFI1 expression was improved compared with patients with low GFI1 expression [34]. The trend in the results of the studies above is similar to our findings for GFI1 in right-sided colon cancer. Here, our main purpose is to identify prognostic factors to separately predict left- and right-sided colon cancers. Although these genes have been studied in colon cancer, little has been reported regarding their ability to predict prognosis, no relevant researches have pointed out that these prognosis-related genes were associated with drugs treatment; thus, the results of this study still need to be verified with data from more samples.

In addition, CCL5, GNG4, GNLY, GZMH, DRD2, and FASLG play a central role among all the DEGs through PPI analysis. Accumulating evidence has clearly demonstrated that CCL5 plays a pivotal part in the progress of CRC [35]. CCL5, a crucial issue being involved in immune escape, it can raise the buildup of myeloid-derived suppressor cells and T-regulatory cells in the course of CRC. Hence, CCL5 is essential for intermediating regulative impacts in the process of CRC [36]. Tumor necrosis factor (TNF)- $\alpha$-preactivated hMSCs exude high levels of CCL5 and then aid the progress of CRC by activation of the epithelial-mesenchymal transition (EMT) process [37]. GNG4 is one of the most hypermethylated and down-regulated genes in glioblastoma. Overexpression of GNG4 inhibits cell growth of glioblastoma cell lines and in vitro transformation of immortalized human astrocytes, hence indicating a probable tumor suppressor character of GNG4 in glioblastoma [38]. Palma P et al. identified increased GNG4 expression level in treatment-responsive rectal cancer samples compared with nonresponsive rectal tumor patients [30]. Our results reveled that GNG4 is highly expressed in 
the left-side colon. The tumor microenvironment comprises a multiplex network of immune T-cell subpopulations (relationship between tumor microenvironment and immunity). Camus $\mathrm{M}$ et al. demonstrated that the coexpression of GNLY and IRF1 could precisely forecast patient survival independent of the situation of metastasis [39]. GZMH, a functional cytotoxic serine protease of NK cell granules, could expand the cell death-inducing repertoire of the innate immune system. GZMH expression was reduced in breast cancer patients compared with that in healthy women [40]. Gemignani $\mathrm{F}$ et al. pointed to a possible role of the dopamine receptor DRD2 in modulating the risk of colorectal cancer [41]. Fas ligand (FasL, CD95L, or TNFSF6) primarily activated T cells, NKT cells, and NK cells [42]. CD95L is a critical survival factor for cancer cells and protects and promotes cancer stem cells [43]. Raats DA et al. concluded that FASLG prompts senescence in mismatch repair-deficient human colon cancer through chronic caspase-mediated induction of DNA damage [44]. Therefore, these core genes play different roles in the occurrence and development of colon cancer through different mechanisms.

Using GSEA, we found that the right colon is closely related to the pathways of antigen processing and presentation, natural killer cell mediated cytotoxicity, and intestinal immune network for IgA production; therefore, there may be differences in the immune mechanisms between left- and right-sided colon cancers. Hypoxia-induced autophagy boosts antigen presentation in the inflammatory response [45]. Lai MC et al. demonstrated that hypoxia upregulated the translation of genes involved in antigen processing and presentation in colon cancer cells [46]. Natural killer cell-mediated cytotoxicity is related to the process of earlyonset CRC and involved in immunosuppression and the epithelial-mesenchymal transition (EMT) in CRC patients [47]. However, differences in the immunological mechanisms of leftand right-sided colon cancers still need to be further explored.

With the development of high-throughput technologies and the improvement of various databases [14, 48-53], some studies have demonstrated that the molecular biological characteristics of left- and right-sided colon cancers impact prognosis. Chromosomal instability (CIN) is one of the manifestations that may cause inactivation of the tumor suppressor gene p53 and is considered an independent prognostic factor for colon cancer [54]. Another study also found that the incidence of p53 mutations in left-sided colon cancer was increased compared with that in right-sided colon cancer. Cases with p53 mutations are at increased risk of death [55]. Microsatellite instability (MSI) is highly associated with rightsided colon cancer [56]. Popat S et al. confirmed that MSI is one of the independent factors that can accurately predict prognosis [57]. The CpG island hypermethylation phenotype (CIMP) often leads to silencing of tumor suppressor genes and tumorigenesis. Iacopetta B found that CIMP often occurred in the right-sided colon [58]. Barault et al. reported that the 5 -year survival rate of the patients with a MSI stable genotype and positive for CIMP was poor. Juo et al. found that CIMP is an independent risk factor for poor prognosis in colorectal cancer [59]. This finding indicates that low expression of the genes in right-sided colon cancer is possibly related to hypermethylation. The RAS family is a family of key effector molecules which is downstream of EGFR, and the incidence of EGFR activation via RAS mutations in right-sided colon cancer is significantly increased as compared to the left-sided colon [60]. K-RAS mutations are associated with poor prognosis because patients with these mutations cannot benefit from chemotherapy [61]. BRAF is also a pivotal molecule in the MARK signaling pathway. Studies found that the incidence of BRAF mutations in right-sided colon cancer was increase compared with that in left-sided colon cancer [62,63]. Correlation meta-analysis revealed that BRAF mutation is a high risk factor for the prognosis of colon cancer [64]. The difference in genetic alterations between left- and the right-sided colon cancers should be further studied.

Our study still has some limitations. We tried to examine the prognostic value of the current model with several genes by the microarray data from GEO database (GSE14333, GSE37892 and GSE39084). But unfortunately those prognostic genes identified by our study showed no prognostic value in GEO data. We consider that the inconsistence may be due to the following reasons. First, sample heterogeneities such as countries, race, and accurate

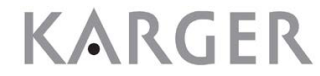




\section{Cellular Physiology Cell Physiol Biochem 2018:48:475-490 \begin{tabular}{l|l} 
and Biochemistry Published online: July 17, 2018 & $\begin{array}{l}\text { C) } 2018 \text { The Author(s). Published by S. Karger AG, Basel } \\
\text { www.karger.com/cpb }\end{array}$
\end{tabular} \\ Liang et al.: Prognostic Signatures in Left- and Right-Side Colon Cancer}

tumor position could cause altered results. Second, these independent GSE studies only contained limited number of samples to generate precise results. We will utilize clinical samples of our hospital or other independent cohorts for verification in the future.

In conclusion, given differences in clinical practice, molecular biological characteristics, survival, and prognosis between left- and right-sided colon cancers, these cancers can be interpreted as two different diseases. In this study, left- and right-sided colon cancer cases were investigated based on gene expression in the TCGA database to clarify their differences and construct separate prognostic models, providing the basis for the individualized treatment and prognosis assessment of left- and right-sided colon cancers.

\section{Acknowledgements}

This study is supported by the Guangxi Medical University Training Program for Distinguished Young Scholars (2017) and Medical Excellence Award Funded by the Creative Research Development Grant from the First Affiliated Hospital of Guangxi Medical University. It was also supported by the public databases of The Cancer Genome Atlas (TCGA), Surveillance Epidemiology and Results (SEER) and Gene Expression Omnibus (GEO).

\section{Disclosure Statement}

The authors declare to have no competing interests.

\section{References}

1 Siegel RL, Miller KD, Jemal A: Cancer Statistics, 2017 CA Cancer J Clin 2017;67:7-30.

-2 Siegel RL, Miller KD, Fedewa SA, Ahnen DJ, Meester RGS, Barzi A, Jemal A: Colorectal cancer statistics, 2017 CA Cancer J Clin 2017;67:177-193.

3 Zeng JH, Liang L, He RQ, Tang RX, Cai XY, Chen JQ, Luo DZ, Chen G: Comprehensive investigation of a novel differentially expressed lncRNA expression profile signature to assess the survival of patients with colorectal adenocarcinoma. Oncotarget 2017;8:16811-16828.

4 Chen H, Yang X, Feng Z, Tang R, Ren F, Wei K, Chen G: Prognostic value of Caspase-3 expression in cancers of digestive tract: a meta-analysis and systematic review. Int J Clin Exp Med 2015;8:10225-10234.

5 Huang L, Ren F, Tang R, Feng Z, Chen G: Prognostic Value of Expression of Cyclin E in Gastrointestinal Cancer: A Systematic Review and Meta-Analysis. Technol Cancer Res Treat 2016;15:12-19.

-6 Bufill JA: Colorectal cancer: evidence for distinct genetic categories based on proximal or distal tumor location. Ann Intern Med 1990;113:779-788.

7 Weiss JM, Pfau PR, O'Connor ES, King J, LoConte N, Kennedy G, Smith MA: Mortality by stage for rightversus left-sided colon cancer: analysis of surveillance, epidemiology, and end results--Medicare data. J Clin Oncol 2011;29:4401-4409.

-8 Pritchard CC, Grady WM: Colorectal cancer molecular biology moves into clinical practice. Gut 2011;60:116-129.

-9 Markowitz SD, Bertagnolli MM: Molecular origins of cancer: Molecular basis of colorectal cancer. N Engl J Med 2009;361:2449-2460.

10 Cheng L, Eng C, Nieman LZ, Kapadia AS, Du XL: Trends in colorectal cancer incidence by anatomic site and disease stage in the United States from 1976 to 2005 Am J Clin Oncol 2011;34:573-580.

11 Yahagi M, Okabayashi K, Hasegawa H, Tsuruta M, Kitagawa Y: The Worse Prognosis of Right-Sided Compared with Left-Sided Colon Cancers: a Systematic Review and Meta-analysis. J Gastrointest Surg 2016;20:648-655.

-12 Moretto R, Cremolini C, Rossini D, Pietrantonio F, Battaglin F, Mennitto A, Bergamo F, Loupakis F, Marmorino F, Berenato R, Marsico VA, Caporale M, Antoniotti C, Masi G, Salvatore L, Borelli B, Fontanini G, Lonardi S, De Braud F, Falcone A: Location of Primary Tumor and Benefit From Anti-Epidermal Growth Factor Receptor Monoclonal Antibodies in Patients With RAS and BRAF Wild-Type Metastatic Colorectal Cancer. Oncologist 2016;21:988-994. 


\section{Cellular Physiology Cell Physiol Biochem 2018;48:475-490 \begin{tabular}{l|l} 
DOI: 10.1159/000491778 & O 2018 The Author(s). Published by S. Karger AG, Basel \\
www.karger.com/cpb
\end{tabular}

13 Benson AB, 3rd, Venook AP, Cederquist L, Chan E, Chen YJ, Cooper HS, Deming D, Engstrom PF, Enzinger PC, Fichera A, Grem JL, Grothey A, Hochster HS, Hoffe S, Hunt S, Kamel A, Kirilcuk N, Krishnamurthi S, Messersmith WA, Mulcahy MF, Murphy JD, Nurkin S, Saltz L, Sharma S, Shibata D, Skibber JM, Sofocleous CT, Stoffel EM, Stotsky-Himelfarb E, Willett CG, Wu CS, Gregory KM, Freedman-Cass D: Colon Cancer, Version 1.2017, NCCN Clinical Practice Guidelines in Oncology. J Natl Compr Canc Netw 2017;15:370-398.

14 Slattery ML, Pellatt DF, Mullany LE, Wolff RK, Herrick JS: Gene expression in colon cancer: A focus on tumor site and molecular phenotype. Genes Chromosomes Cancer 2015;54:527-541.

15 Barras D, Missiaglia E, Wirapati P, Sieber OM, Jorissen RN, Love C, Molloy PL, Jones IT, McLaughlin S, Gibbs P, Guinney J, Simon IM, Roth AD, Bosman FT, Tejpar S, Delorenzi M: BRAF V600E Mutant Colorectal Cancer Subtypes Based on Gene Expression. Clin Cancer Res 2017;23:104-115.

16 Shen S, Bai J, Wei Y, Wang G, Li Q, Zhang R, Duan W, Yang S, Du M, Zhao Y, Christiani DC, Chen F: A sevengene prognostic signature for rapid determination of head and neck squamous cell carcinoma survival. Oncol Rep 2017;38:3403-3411.

17 He RQ, Wei QJ, Tang RX, Chen WJ, Yang X, Peng ZG, Hu XH, Ma J, Chen G: Prediction of clinical outcome and survival in soft-tissue sarcoma using a ten-lncRNA signature. Oncotarget 2017;8:80336-80347.

18 Guo L, Zhang K, Bing Z: Application of a coexpression network for the analysis of aggressive and nonaggressive breast cancer cell lines to predict the clinical outcome of patients. Mol Med Rep 2017;16:7967-7978.

19 Luo YH, Liang L, He RQ, Wen DY, Deng GF, Yang H, He Y, Ma W, Cai XY, Chen JQ Chen G: RNA-sequencing investigation identifies an effective risk score generated by three novel lncRNAs for the survival of papillary thyroid cancer patients. Oncotarget 2017;8:74139-74158.

20 Wang Z, Wu Q, Feng S, Zhao Y, Tao C: Identification of four prognostic LncRNAs for survival prediction of patients with hepatocellular carcinoma. PeerJ 2017;5:e3575.

-21 Zhou X, Huang Z, Xu L, Zhu M, Zhang L, Zhang H, Wang X, Li H, Zhu W, Shu Y, Liu P: A panel of 13-miRNA signature as a potential biomarker for predicting survival in pancreatic cancer. Oncotarget 2016;7:6961669624.

-22 Subramanian A, Tamayo P, Mootha VK, Mukherjee S, Ebert BL, Gillette MA, Paulovich A, Pomeroy SL, Golub TR, Lander ES, Mesirov JP: Gene set enrichment analysis: a knowledge-based approach for interpreting genome-wide expression profiles. Proc Natl Acad Sci U S A 2005;102:15545-15550.

-23 Bauer KM, Hummon AB, Buechler S: Right-side and left-side colon cancer follow different pathways to relapse. Mol Carcinog 2012;51:411-421.

24 Bauer KM, Watts TN, Buechler S, Hummon AB: Proteomic and functional investigation of the colon cancer relapse-associated genes NOX4 and ITGA3 J Proteome Res 2014;13:4910-4918.

25 Itoh A, Uchiyama A, Taniguchi S, Sagara J: Phactr3/scapinin, a member of protein phosphatase 1 and actin regulator (phactr) family, interacts with the plasma membrane via basic and hydrophobic residues in the N-terminus. PLoS One 2014;9:e113289.

-26 Bosch LJ, Oort FA, Neerincx M, Khalid-de Bakker CA, Terhaar sive Droste JS, Melotte V, Jonkers DM, Masclee AA, Mongera S, Grooteclaes M, Louwagie J, van Criekinge W, Coupe VM, Mulder CJ, van Engeland M, Carvalho B, Meijer GA: DNA methylation of phosphatase and actin regulator 3 detects colorectal cancer in stool and complements FIT. Cancer Prev Res (Phila) 2012;5:464-472.

-27 Rasmussen SL, Krarup HB, Sunesen KG, Pedersen IS, Madsen PH, Thorlacius-Ussing O: Hypermethylated DNA as a biomarker for colorectal cancer: a systematic review. Colorectal Dis 2016;18:549-561.

28 Wang H, Tang M, Ou L, Hou M, Feng T, Huang YE, Jin Y, Zhang H, Zuo G: Biological analysis of cancer specific microRNAs on function modeling in osteosarcoma. Sci Rep 2017;7:5382.

29 Saridaki Z, Tzardi M, Papadaki C, Sfakianaki M, Pega F, Kalikaki A, Tsakalaki E, Trypaki M, Messaritakis I, Stathopoulos E, Mavroudis D, Georgoulias V, Souglakos J: Impact of KRAS, BRAF, PIK3CA mutations, PTEN, AREG, EREG expression and skin rash in $>/=2$ line cetuximab-based therapy of colorectal cancer patients. PLoS One 2011;6:e15980.

30 Qu X, Sandmann T, Frierson H, Jr., Fu L, Fuentes E, Walter K, Okrah K, Rumpel C, Moskaluk C, Lu S, Wang Y, Bourgon R, Penuel E, Pirzkall A, Amler L, Lackner MR, Tabernero J, Hampton GM, Kabbarah O: Integrated genomic analysis of colorectal cancer progression reveals activation of EGFR through demethylation of the EREG promoter. Oncogene 2016;35:6403-6415. 


\section{Cellular Physiology Cell Physiol Biochem 2018;48:475-490 \begin{tabular}{l|l} 
DOI: 10.1159/000491778 & O 2018 The Author(s). Published by S. Karger AG, Basel \\
www.karger.com/cpb
\end{tabular}}

Liang et al.: Prognostic Signatures in Left- and Right-Side Colon Cancer

-31 Lee MS, McGuffey EJ, Morris JS, Manyam G, Baladandayuthapani V, Wei W, Morris VK, Overman MJ, Maru DM, Jiang ZQ, Hamilton SR, Kopetz S: Association of CpG island methylator phenotype and EREG/AREG methylation and expression in colorectal cancer. Br J Cancer 2016;114:1352-1361.

-32 Inoue A, Okamoto K, Fujino Y, Nakagawa T, Muguruma N, Sannomiya K, Mitsui Y, Takaoka T, Kitamura S, Miyamoto H, Okahisa T, Fujimori T, Imoto I, Takayama T: B-RAF mutation and accumulated gene methylation in aberrant crypt foci (ACF), sessile serrated adenoma/polyp (SSA/P) and cancer in SSA/P. Br J Cancer 2015;112:403-412.

-33 Zou H, Hu L, Li J, Zhan S, Cao K: Cloning and characterization of a novel small monomeric GTPase, RasL10B, with tumor suppressor potential. Biotechnol Lett 2006;28:1901-1908.

34 Xing W, Xiao Y, Lu X, Zhu H, He X, Huang W, Lopez ES, Wong J, Ju H, Tian L, Zhang F, Xu H, Wang SD, Li X, Karin M, Ren H: GFI1 downregulation promotes inflammation-linked metastasis of colorectal cancer. Cell Death Differ 2017;24:929-943.

-35 Kan JY, Wu DC, Yu FJ, Wu CY, Ho YW, Chiu YJ, Jian SF, Hung JY, Wang JY, Kuo PL: Chemokine (C-C Motif) Ligand 5 is Involved in Tumor-Associated Dendritic Cell-Mediated Colon Cancer Progression Through NonCoding RNA MALAT-1 J Cell Physiol 2015;230:1883-1894.

-36 Zhang Y, Lv D, Kim HJ, Kurt RA, Bu W, Li Y, Ma X: A novel role of hematopoietic CCL5 in promoting triplenegative mammary tumor progression by regulating generation of myeloid-derived suppressor cells. Cell Res 2013;23:394-408.

-37 Chen K, Liu Q, Tsang LL, Ye Q, Chan HC, Sun Y, Jiang X: Human MSCs promotes colorectal cancer epithelial-mesenchymal transition and progression via CCL5/beta-catenin/Slug pathway. Cell Death Dis 2017;8:e2819.

38 Pal J, Patil V, Mondal B, Shukla S, Hegde AS, Arivazhagan A, Santosh V, Somasundaram K: Epigenetically silenced GNG4 inhibits SDF1alpha/CXCR4 signaling in mesenchymal glioblastoma. Genes Cancer 2016;7:136-147.

-39 Camus M, Tosolini M, Mlecnik B, Pages F, Kirilovsky A, Berger A, Costes A, Bindea G, Charoentong P, Bruneval P, Trajanoski Z, Fridman WH, Galon J: Coordination of intratumoral immune reaction and human colorectal cancer recurrence. Cancer Res 2009;69:2685-2693.

-40 Tahbaz-Lahafi B, Akbari ME, Amir-Rassouli H, Rahimipour A, Azargashb E, Nafissi N, Jahani F: Granzyme $\mathrm{h}$ serum levels variations with both reproductive hormone receptors, and related hormone receptors in breast cancer patients. Iran J Cancer Prev 2014;7:48-52.

41 Nagata S: Fas ligand-induced apoptosis. Annu Rev Genet 1999;33:29-55.

42 Aggarwal BB: Signalling pathways of the TNF superfamily: a double-edged sword. Nat Rev Immunol 2003;3:745-756.

43 Peter ME, Hadji A, Murmann AE, Brockway S, Putzbach W, Pattanayak A, Ceppi P: The role of CD95 and CD95 ligand in cancer. Cell Death Differ 2015;22:549-559.

44 Raats DA, Frenkel N, van Schelven SJ, Rinkes IH, Laoukili J, Kranenburg O: CD95 ligand induces senescence in mismatch repair-deficient human colon cancer via chronic caspase-mediated induction of DNA damage. Cell Death Dis 2017;8:e2669.

45 Ye J, Gao Z, Yin J, He Q: Hypoxia is a potential risk factor for chronic inflammation and adiponectin reduction in adipose tissue of ob/ob and dietary obese mice. Am J Physiol Endocrinol Metab 2007;293:E1118-1128.

-46 Lai MC, Chang CM, Sun HS: Hypoxia Induces Autophagy through Translational Up-Regulation of Lysosomal Proteins in Human Colon Cancer Cells. PLoS One 2016;11:e0153627.

47 Nam S, Park T: Pathway-based evaluation in early onset colorectal cancer suggests focal adhesion and immunosuppression along with epithelial-mesenchymal transition. PLoS One 2012;7:e31685.

48 Liu L, Li L, Ma X, Yue F, Wang Y, Wang L, Jin P, Zhang X: Altered Circular RNA Expression in Patients with Repeated Implantation Failure. Cell Physiol Biochem 2017;44:303-313.

49 Sui J, Li YH, Zhang YQ, Li CY, Shen X, Yao WZ, Peng H, Hong WW, Yin LH, Pu YP, Liang GY: Integrated analysis of long non-coding RNAassociated ceRNA network reveals potential lncRNA biomarkers in human lung adenocarcinoma. Int J Oncol 2016;49:2023-2036.

50 Han D, Chen Y, Kou Y, Weng J, Chen B, Yu Y, Zhang P, Jiang B: Profiling of the dynamically alteredgene expression in peripheral nerve injury using NGS RNA sequencing technique. Am J Transl Res 2016;8:871884. 


\section{Cellular Physiology Cell Physiol Biochem 2018;48:475-490 \begin{tabular}{l|l} 
and Biochemistry Published online: July 17, 2018 & $\begin{array}{l}\text { D) } 2018 \text { The Author(s). Published by S. Karger AG, Basel } \\
\text { www.karger.com/cpb }\end{array}$ \\
\hline
\end{tabular}}

Liang et al.: Prognostic Signatures in Left- and Right-Side Colon Cancer

51 Yang J, Zhang P, Wang L: Gene Network for Identifying the Entropy Changes of Different Modules in Pediatric Sepsis. Cell Physiol Biochem 2016;40:1153-1162.

52 Rooprai HK, Martin AJ, King A, Appadu UD, Jones H, Selway RP, Gullan RW, Pilkington GJ: Comparative gene expression profiling of ADAMs, MMPs, TIMPs, EMMPRIN, EGF-R and VEGFA in low grade meningioma. Int J Oncol 2016;49:2309-2318.

53 Li H, Hao X, Wang H, Liu Z, He Y, Pu M, Zhang H, Yu H, Duan J, Qu S: Circular RNA Expression Profile of Pancreatic Ductal Adenocarcinoma Revealed by Microarray. Cell Physiol Biochem 2016;40:1334-1344.

54 Gao P, Song YX, Xu YY, Sun Z, Sun JX, Xu HM, Wang ZN: Does the prognosis of colorectal mucinous carcinoma depend upon the primary tumour site? Results from two independent databases. Histopathology 2013;63:603-615.

55 Russo A, Bazan V, Iacopetta B, Kerr D, Soussi T, Gebbia N, Group TCCS: The TP53 colorectal cancer international collaborative study on the prognostic and predictive significance of p53 mutation: influence of tumor site, type of mutation, and adjuvant treatment. J Clin Oncol 2005;23:7518-7528.

-56 Shin US, Cho SS, Moon SM, Park SH, Jee SH, Jung EJ, Hwang DY: Is microsatellite instability really a good prognostic factor of colorectal cancer? Ann Coloproctol 2014;30:28-34.

57 Popat S, Hubner R, Houlston RS: Systematic review of microsatellite instability and colorectal cancer prognosis. J Clin Oncol 2005;23:609-618.

58 Iacopetta B, Heyworth J, Girschik J, Grieu F, Clayforth C, Fritschi L: The MTHFR C677T and DeltaDNMT3B C-149T polymorphisms confer different risks for right- and left-sided colorectal cancer. Int J Cancer 2009;125:84-90.

59 Juo YY, Johnston FM, Zhang DY, Juo HH, Wang H, Pappou EP, Yu T, Easwaran H, Baylin S, van Engeland M, Ahuja N: Prognostic value of CpG island methylator phenotype among colorectal cancer patients: a systematic review and meta-analysis. Ann Oncol 2014;25:2314-2327.

-60 Tong JH, Lung RW, Sin FM, Law PP, Kang W, Chan AW, Ma BB, Mak TW, Ng SS, To KF: Characterization of rare transforming KRAS mutations in sporadic colorectal cancer. Cancer Biol Ther 2014;15:768-776.

-61 Ogura T, Kakuta M, Yatsuoka T, Nishimura Y, Sakamoto H, Yamaguchi K, Tanabe M, Tanaka Y, Akagi K: Clinicopathological characteristics and prognostic impact of colorectal cancers with NRAS mutations. Oncol Rep 2014;32:50-56.

62 Yamauchi M, Morikawa T, Kuchiba A, Imamura Y, Qian ZR, Nishihara R, Liao X, Waldron L, Hoshida Y, Huttenhower C, Chan AT, Giovannucci E, Fuchs C, Ogino S: Assessment of colorectal cancer molecular features along bowel subsites challenges the conception of distinct dichotomy of proximal versus distal colorectum. Gut 2012;61:847-854.

63 Smith CG, Fisher D, Claes B, Maughan TS, Idziaszczyk S, Peuteman G, Harris R, James MD, Meade A, Jasani B, Adams RA, Kenny S, Kaplan R, Lambrechts D, Cheadle JP: Somatic profiling of the epidermal growth factor receptor pathway in tumors from patients with advanced colorectal cancer treated with chemotherapy +/cetuximab. Clin Cancer Res 2013;19:4104-4113.

64 Eklof V, Wikberg ML, Edin S, Dahlin AM, Jonsson BA, Oberg A, Rutegard J, Palmqvist R: The prognostic role of KRAS, BRAF, PIK3CA and PTEN in colorectal cancer. Br J Cancer 2013;108:2153-2163. 